\title{
Simple Rational Polynomials and the Jacobian Conjecture
}

\author{
in honour of Professor Heisuke Hironaka
}

By

\author{
LÊ Dũng Tráng*
}

\section{Introduction}

Consider an algebraic map $\Phi:=(f, g): \mathbb{C}^{2} \rightarrow \mathbb{C}^{2}$ defined by the polynomial functions $f$ and $g$ of $\mathbb{C}^{2}$. In complex dimension 2 the Jacobian conjecture asserts that, if the determinant $J(f, g)$ of the Jacobian matrix of $\Phi$ is a nonzero constant, then the algebraic map $\Phi$ is an isomorphism.

In this paper we shall show that this conjecture is true, when we assume that one of the two polynomial functions has generic rational fibers, i.e. they are diffeomorphic to a punctured 2-sphere, and furthermore is simple, i.e. the dicritical components of the polynomial in a natural compactification have degree one (see [10]). We call rational polynomial functions polynomial functions which have a general fiber diffeomorphic to a punctured 2-sphere. The corresponding polynomials are called rational polynomials. In [10] one can find a classification of simple rational polynomials up to right-left equivalence, but we shall not use this classification to establish our result.

\section{$\S 1 . \quad$ Rational Polynomials}

$\S 1.1$.

In all this paper we shall only consider complex polynomial functions from $\mathbb{C}^{2}$ into $\mathbb{C}$. We denote in the same way a complex polynomial function

\footnotetext{
Communicated by K. Saito. Received March 12, 2007. Revised September 17, 2007.

2000 Mathematics Subject Classification(s): 14R15, 14H20, $14 \mathrm{~J} 17$.

*The "Abdus Salam" ICTP, Strada Costiera 11, 34014 Trieste, Italy.

e-mail: ledt@ictp.it
}

(c) 2008 Research Institute for Mathematical Sciences, Kyoto University. All rights reserved. 
$f: \mathbb{C}^{2} \rightarrow \mathbb{C}$ and the corresponding polynomial $f \in \mathbb{C}[X, Y]$. Let $f$ be a complex polynomial of degree $d$. We have

$$
f(X, Y)=\sum_{\alpha+\beta \leq d} a_{\alpha, \beta} X^{\alpha} Y^{\beta}
$$

with $a_{\alpha, \beta} \in \mathbb{C}$. The corresponding homogeneized polynomial is:

$$
F(X, Y, T)=\sum_{\alpha+\beta \leq d} a_{\alpha, \beta} X^{\alpha} Y^{\beta} T^{d-\alpha-\beta} .
$$

This defines a rational function $F / T^{d}: \mathbb{P}^{2}--\rightarrow \mathbb{P}^{1}$ given by

$$
F / T^{d}(X: Y: T):=\left(F(X, Y, T): T^{d}\right) .
$$

The set of indeterminacy points of $F / T^{d}$ contained in the line at infinity $T=0$ of $\mathbb{C}^{2}$ is the set of asymptotic directions $\left(x_{i}: y_{i}: 0\right)$ of $f$ such that

$$
f_{d}\left(x_{i}, y_{i}\right)=\sum_{\alpha+\beta=d} a_{\alpha, \beta} x_{i}^{\alpha} y_{i}^{\beta}=0 .
$$

It is well-known (see e.g. [8]) that after a modification $\pi: Z \rightarrow \mathbb{P}^{2}$ of $\mathbb{P}^{2}$, composition of a sequence of point blowing-ups, over the indeterminacy points of $F / T^{d}$, we have an algebraic map $\varphi: Z \rightarrow \mathbb{P}^{1}$ which extends $F / T^{d}$, i.e. for any point $m$ of $\mathbb{P}^{2}$ different of the indeterminacy points of $F / T^{d}$, we have $F / T^{d}(m)=\varphi\left(\pi^{-1}(m)\right)$.

Definition 1.1. A sequence of point blowing-ups $\pi: Z \rightarrow \mathbb{P}^{2}$, such that $F / T^{d}$ extends to an algebraic map $\varphi: Z \rightarrow \mathbb{P}^{1}$, is said to lift the indeterminacy of the rational function $F / T^{d}$.

Let $\pi: Z \rightarrow \mathbb{P}^{2}$ be a sequence of point blowing-ups which lifts the indeterminacy of the rational function $F / T^{d}$. Let $\mathcal{D}$ be the divisor $\pi^{-1}(T=0)$. We call $\mathcal{D}$ the divisor at infinity of $Z$. Of course, the morphism $\pi$ induces an isomorphism of $Z \backslash \mathcal{D}$ over $\mathbb{P}^{2} \backslash\{T=0\}=\mathbb{C}^{2}$.

Let $D_{\infty}$ be the strict transform of $T=0$ by $\pi$. It is a component of $\mathcal{D}$.

A component of $D$ of $\mathcal{D}$ is one of three types:

1. The restriction of $\varphi$ to $D$ is a non-constant map over $\mathbb{P}^{1}$;

2. The restriction of $\varphi$ to $D$ is a constant map with value $\infty:=(1: 0) \in \mathbb{P}^{1}$;

3. The restriction of $\varphi$ to $D$ is a constant map with value $\neq \infty$. 
The components of the type 1 are called dicritical components of $f$ (or $F / T^{d}$ or $\varphi$ ) in $Z$. We shall denote by $\mathcal{D}_{\infty}$ the divisor $\varphi^{-1}(\infty)$. Of course $D_{\infty} \subset \mathcal{D}_{\infty}$.

Using Zariski connectedness theorem, we can prove (see [9] or [7]):

Theorem 1.2. $\quad$ The divisor $\mathcal{D}_{\infty}$ is connected.

Let us define:

Definition 1.3. A modification $\pi: Z \rightarrow \mathbb{P}^{2}$ of $\mathbb{P}^{2}$ which lifts the indeterminacy of a rational function $F / T^{d}$ is called minimal if the only components of the divisor $\mathcal{D}:=\pi^{-1}(T=0)$ which have self-intersection -1 are among the dicritical components of $F / T^{d}$ or $D_{\infty}$, the strict transform by $\pi$ of $T=0$.

One can prove that any modification

$$
\pi: Z \rightarrow \mathbb{P}^{2}
$$

which lifts the indeterminacy of a rational function $F / T^{d}$ factorizes uniquely through a given minimal one. This shows that a minimal sequence of point blowing-ups which lifts the indeterminacy of the rational function $F / T^{d}$ is unique up to algebraic isomorphism.

In [8] (see also [7]) we have observed the following result:

Theorem 1.4. a) For all sequence of point blowing-ups $\pi: Z \rightarrow \mathbb{P}^{2}$ which lifts the indeterminacy of a rational function $F / T^{d}$, the intersection graph of the divisor $\mathcal{D}:=\pi^{-1}(T=0)$ is a tree.

b) Let a minimal sequence of point blowing-ups $\pi: Z \rightarrow \mathbb{P}^{2}$ which lifts the indeterminacy of a rational function $F / T^{d}$. Consider the finite set $\left(\mathcal{A}_{k}\right)_{k \in K}$ of closures of connected components of $\mathcal{D} \backslash \mathcal{D}_{\infty}$, then

1. The sets $\mathcal{A}_{k}(k \in K)$ are pairwise distinct.

2. Each $\mathcal{A}_{k}$ contains exactly one dicritical component and this dicritical component contains the intersection point of $\mathcal{A}_{k}$ and $\mathcal{D}_{\infty}$.

3. The intersection graph $\mathcal{B}_{k}$ of $\mathcal{A}_{k}$ is a bamboo, i.e. a graph which is a linear chain, and one of its extremity is the dicritical component $D_{k}$. In particular the intersection graph of the closure of $\mathcal{A}_{k} \backslash D_{k}$ is a sub-bamboo $\mathcal{B}_{k}^{\prime}$ of $\mathcal{B}_{k}$ which might be empty in some cases. 


\section{$\S 1.2$.}

We shall consider complex rational polynomial functions.

Definition 1.5. A complex polynomial function $f: \mathbb{C}^{2} \rightarrow \mathbb{C}$ is said to be rational if its general fiber is a rational curve, i.e. diffeomorphic to a punctured 2-sphere.

As above one can consider the homogeneisation $F$ of the rational polynomial $f$ and the rational function $F / T^{d}$ that it defines on $\mathbb{P}^{2}$. Let $\pi: Z \rightarrow \mathbb{P}^{2}$ be a sequence of point blowing-ups over the asymptotic directions of $f$ which lifts the indeterminacy of $F / T^{d}$ and defines the map $\varphi: Z \rightarrow \mathbb{P}^{1}$ which extends the polynomial function $f$. A classical result (see e.g. [4, p. 521]) shows that:

Proposition 1.6. $\quad$ There exists a composition of a finite sequence of contractions on points $q: Z \rightarrow Z_{1}$ and a locally trivial fibration $\varphi_{1}: Z_{1} \rightarrow \mathbb{P}^{1}$, such that $\varphi_{1} \circ q=\varphi$.

We have the following corollaries:

Corollary 1.7. The fibers of a complex rational polynomial are normal crossing divisors of non-singular rational curves.

Proof. By assumption, this is already true for general fibers. Let $f=\lambda$ be a fiber which is not general. The fiber $\varphi^{-1}(\lambda)$ retracts on the rational curve $\varphi_{1}^{-1}(\lambda)$, so it is a normal crossing divisor whose components are all non-singular rational curves. This easily implies our result.

Corollary 1.8. Let $f: \mathbb{C}^{2} \rightarrow \mathbb{C}$ be a rational polynomial function and consider a minimal modification $\pi: Z \rightarrow \mathbb{P}^{2}$ which lifts the indeterminacy of the corresponding rational function $F / T^{d}$. If the degree of the polynomial $f$ is $\geq 2$, the strict transform $D_{\infty}$ of the line at infinity $T=0$ has self-intersection -1 .

Proof. According to the preceding proposition 1.6 there is a finite sequence of contractions on points $q: Z \rightarrow Z_{1}$ and a locally trivial fibration $\varphi_{1}: Z_{1} \rightarrow \mathbb{P}_{1}$, such that $\varphi_{1} \circ q=\varphi$, so the divisor $\mathcal{D}_{\infty}:=\varphi^{-1}(\infty)$ contracts onto $\varphi_{1}^{-1}(\infty)$. Since the morphism $\pi$ is a minimal sequence of point blowingups which lifts the indeterminacy of $F / T^{d}$, the only possible component of $\mathcal{D}_{\infty}$ which might have self-intersection -1 and therefore might contract is $D_{\infty}$. If the degree of $f$ is $\geq 2$, the morphism $q$ is not an isomorphism, so necessarily $D_{\infty}$ has self-intersection -1 . 


\section{$\S 1.3 . \quad$ Examples}

Let $(f, g)$ be an automorphism of $\mathbb{C}^{2}$. Obviously $f$ and $g$ are rational polynomials. In the class of rational polynomials they are particular rational polynomials. For instance, they are locally trivial fibrations over $\mathbb{C}$. Their compactifications have been studied in [3].

Consider $f(X, Y)=X(X Y-1)$. It is a polynomial of degree 3. For $\lambda \neq 0$ the fiber $f^{-1}(\lambda)$ is general and diffeomorphic to a cylinder, i.e. to a 2 -sphere minus two distinct points, the asymptotic directions of $f$. Obviously $f^{-1}(0)$ is the union of a line and a cylinder given by $X Y=1$. So, $f$ is a rational polynomial. We observe that it is not a locally trivial fibration over $\mathbb{C}$.

\section{§2. Rational Polynomials in Jacobian Pairs}

Let us call Jacobian pair a pair $(f, g)$ of polynomial functions on $\mathbb{C}^{2}$ such that the determinant of the Jacobian matrix $J(f, g)$ is a non-zero constant.

In this section we shall consider rational polynomials $f$ which belong to a Jacobian pair $(f, g)$.

\section{$\S 2.1 . \quad$ Finite fibers}

A rational polynomial function which belongs to a Jacobian pair has no critical points. This implies that all the fibers of such rational polynomials are non-singular (in particular reduced). We have the following theorem:

Theorem 2.1. $\quad$ Let $f$ be a rational polynomial function of $\mathbb{C}^{2}$. Assume that $f$ has no critical point. Let $\pi: Z \rightarrow \mathbb{P}^{2}$ be a minimal sequence of point blowing-ups which lifts the indeterminacy of the corresponding rational function $F / T^{d}$. Let $\varphi$ the extension of $f$ to $Z$. The fibers $\varphi^{-1}(\lambda)$ for $\lambda \neq \infty$ are reduced normal crossing divisors.

Proof. If $\lambda$ is a general value of $f$, our result is obvious. Consider an atypical value $\lambda$ of $f$. If $\varphi^{-1}(\lambda)$ does not contain components of $\mathcal{D}$, we have $f^{-1}(\lambda)=\varphi^{-1}(\lambda) \backslash \cup_{k \in K} D_{k}$, where $\left(D_{k}\right)_{k \in K}$ is the set of dicritical components of $f$. Since $f^{-1}(\lambda)$ is reduced, in this case $\varphi^{-1}(\lambda)$ is also reduced and, by Corollary 1.7 , it is a normal crossing divisor. It remains to consider the case $\varphi^{-1}(\lambda)$ contains components of $\mathcal{D}$.

In this last case, it is easy to see that the components contained in $\varphi^{-1}(\lambda)$ are the components of some of the sub-bamboos $\mathcal{B}_{k}^{\prime}$ given in Theorem 1.4 above. 
The intersection graph of $\varphi^{-1}(\lambda)$ is the tree obtained from such sub-bamboos $\mathcal{B}_{k}^{\prime}$ to which we attach vertices corresponding to the strict transforms by $\pi$ of the closures of the components of $f^{-1}(\lambda)$. To fix the notations let us call $D_{1}, \ldots, D_{r}$ the components of the sub-bamboos $\mathcal{B}_{k}^{\prime}$ contained in $\varphi^{-1}(\lambda)$ and $\Gamma_{1}, \ldots, \Gamma_{s}$ the strict transforms of the closures of the components of $f^{-1}(\lambda)$. The minimality of $\pi$ implies that the self-intersections of $D_{1}, \ldots, D_{r}$ in $Z$ are $\leq-2$. Among the $\Gamma_{i}$ 's, the ones connecting several sub-bamboos $\mathcal{B}_{k}^{\prime}$ are necessarily of selfintersection $\leq-2$, otherwise they can be contracted and, since they would connect two components of multiplicity $\geq 1$ in $\varphi^{-1}(\lambda)$, they would not be reduced.

To be simple, we shall restrict the proof of the above theorem only to the case the atypical fiber $\varphi^{-1}(\lambda)$ only contains one bamboo $\mathcal{B}_{k}^{\prime}$. We leave the reader to consider the general case by himself.

We shall show that in the case $\varphi^{-1}(\lambda)$ only contains one bamboo $\mathcal{B}_{k}^{\prime}$, we have :

Lemma 2.2. The self-intersections of $\Gamma_{1}, \ldots, \Gamma_{s}$ in $Z$ equal -1 .

Proof. Let $\sum_{i=1}^{i=r} n_{i} D_{i}+\sum_{j=1}^{j=s} \Gamma_{j}$ be the divisor defined by $\varphi^{-1}(\lambda)$ where the components $\Gamma_{j}(1 \leq j \leq s)$ are reduced. Since $\varphi^{-1}(\lambda)$ is a fiber of a function, since $\Gamma_{j}, 1 \leq j \leq s$, are components of $\varphi^{-1}(\lambda)$, we have, for any $j$, $1 \leq j \leq s$

$$
\left(\sum_{i=1}^{i=r} n_{i} D_{i}+\sum_{j=1}^{j=s} \Gamma_{j}\right) \cdot \Gamma_{\ell}=n_{i_{\ell}}+\Gamma_{\ell} \cdot \Gamma_{\ell}=0
$$

where $D_{n_{i_{\ell}}}$ is the component of $\mathcal{B}_{k}^{\prime}$ intersected by $\Gamma_{\ell}$. We have to prove that $n_{i_{\ell}}=1$.

The fiber $\varphi^{-1}(\lambda)$ contracts onto a non-singular rational curve with selfintersection 0 according to the Theorem 1.4 above. Since self-intersections of $D_{1}, \ldots, D_{r}$ in $Z$ are $\leq-2$, at least one of the components $\Gamma_{j}$ has selfintersection -1 in $Z$.

Let us contract $q^{\prime}: Z \rightarrow Z^{\prime}$ all the $\Gamma_{j}, j \in\left\{j_{1}, \ldots, j_{t}\right\}$, which have selfintersection -1 in $Z$. We have a function $\varphi^{\prime}: Z^{\prime} \rightarrow \mathbb{P}^{1}$ such that

$$
\varphi=\varphi^{\prime} \circ q^{\prime}
$$

and $\varphi^{\prime-1}(\lambda)$ is a divisor $\sum_{i=1}^{i=r} n_{i}^{\prime} D_{i}^{\prime}+\sum_{j \in J} \Gamma_{j}^{\prime}$, where $J:=\{1, \ldots, s\} \backslash\left\{j_{1}, \ldots, j_{t}\right\}$. As above, since $D_{i}^{\prime}, 1 \leq i \leq r$, are components of the fiber $\varphi^{\prime-1}(\lambda)$ of the mor- 
phism $\varphi^{\prime}$ in $Z^{\prime}$, for any $i, 1 \leq i \leq r$, we have

$$
\left(\sum_{i=1}^{i=r} n_{i}^{\prime} D_{i}^{\prime}+\sum_{j \in J} \Gamma_{j}^{\prime}\right) \cdot D_{i}^{\prime}=0
$$

which means that

$$
n_{i}^{\prime}\left(D_{i}^{\prime} \cdot D_{i}^{\prime}\right)+\sum_{j \neq i} n_{j}^{\prime} D_{j}^{\prime} \cdot D_{i}^{\prime}+\#\left\{j \in J, \Gamma_{j}^{\prime} \cdot D_{i}^{\prime}=1\right\}=0 .
$$

Since $n_{i}^{\prime}>0, \#\left\{j \neq i, D_{j}^{\prime} \cdot D_{i}^{\prime} \neq 0\right\}=1,2, \#\left\{j \in J, \Gamma_{j}^{\prime} \cdot D_{i}^{\prime}=1\right\} \geq 0$, the self-intersections of the $D_{i}^{\prime}$ in $Z^{\prime}$ are $\leq-1$.

We have to prove that $J=\emptyset$. The intersection graph of $\cup_{i=1}^{i=r} D_{i}^{\prime}$ is a bamboo $\tilde{\mathcal{B}}_{k}^{\prime}$ similar to $\mathcal{B}_{k}^{\prime}$. Since the contraction $q^{\prime}$ involves curves which do not intersect the $\Gamma_{j}, j \in J$, the self-intersections of the $\Gamma_{j}, j \in J$, in $Z$ and of their images by $q^{\prime}$ in $Z^{\prime}$ are equal, so they are $\leq-2$. Since $\varphi^{\prime-1}(\lambda)$ will contract onto a non-singular rational curve with self-intersection 0 , one of the images $D_{i}^{\prime}$ of the $D_{i}$ has self-intersection -1 in $Z^{\prime}$. Such a $D_{i}^{\prime}$ must be an extremity of $\tilde{\mathcal{B}}_{k}^{\prime}$, otherwise, since $D_{i} \cdot D_{i}<D_{i}^{\prime} . D_{i}^{\prime}$, the component $D_{i}$ must intersect components among the $\Gamma_{j}, j \in\left\{j_{1}, \ldots, j_{t}\right\}$ and, on the other hand, this component $D_{i}^{\prime}$, having self-intersection -1 is the exceptional divisor of the blowing-up of the intersection point of components which correspond to adjacent components of $D_{i}$ in $\varphi^{\prime-1}(\lambda)$, so it is a component of multiplicity $n_{i}^{\prime} \geq 2$ in the divisor $\varphi^{\prime-1}(\lambda)$. This would imply that the component $\Gamma_{j}, j \in\left\{j_{1}, \ldots, j_{t}\right\}$, which intersect $D_{i}$ have multiplicity $n_{i}=n_{i}^{\prime} \geq 2$ in $\varphi^{\prime-1}(\lambda)$ which contradicts the fact that $\Gamma_{j}$ is reduced in $\varphi^{-1}(\lambda)$.

Then, a $D_{i}^{\prime}$ with self-intersection -1 is among the extremities of $\tilde{\mathcal{B}}_{k}^{\prime}$. None of the $\Gamma_{j}^{\prime}:=q^{\prime}\left(\Gamma_{j}\right), j \in J$, intersects $D_{i}^{\prime}$, because if one of them $\Gamma_{j_{0}}^{\prime}$ intersects $D_{i}^{\prime}$, since $\Gamma_{j_{0}}^{\prime}$ is a component of the fiber $\varphi^{\prime-1}(\lambda)$, we have:

$$
\left(\sum_{i=1}^{i=r} n_{i}^{\prime} D_{i}^{\prime}+\sum_{j \in J} \Gamma_{j}^{\prime}\right) \cdot \Gamma_{j_{0}}^{\prime}=n_{i}^{\prime}+\Gamma_{j_{0}}^{\prime} \cdot \Gamma_{j_{0}}^{\prime}=0
$$

so, since the self-intersection of $\Gamma_{j_{0}}$ is $\leq-2$ by hypothesis, the multiplicity $n_{i}=n_{i}^{\prime}$ of $D_{i}$ would be $\geq 2$.

Since the self-intersection of $D_{i}^{\prime}$ is -1 , it is $>$ than the self-intersection of $D_{i}$, necessarily there is one of the $\Gamma_{j}, j \in\left\{j_{1}, \ldots, j_{t}\right\}$, which has self-intersection -1 which intersects $D_{i}$. As this $\Gamma_{j}$ is the exceptional divisor of the blowing-up of a point of $D_{i}^{\prime}$, its multiplicity in $\varphi^{-1}(\lambda)$ is $n_{i} \geq 2$. This contradicts the fact that in the fiber $\varphi^{-1}(\lambda)$, it is a reduced component. 
Let us contract $q^{(1)}: Z^{\prime} \rightarrow Z^{(1)}$ all the extremities of $\tilde{\mathcal{B}}_{k}^{\prime}$ which have self-intersection -1 . We also have a function $\varphi^{(1)}: Z^{(1)} \rightarrow \mathbb{P}^{1}$ such that

$$
\varphi^{\prime}=\varphi^{(1)} \circ q^{(1)} .
$$

The image $q^{(1)}\left(\cup_{i=1}^{i=r} D_{i}^{\prime}\right)=\varphi^{(1)^{-1}}(\lambda)$ is a curve $\mathcal{D}^{(1)}$ with non-singular rational components and intersection tree, a bamboo $\mathcal{B}^{(1)}$.

Again one of the extremities of $\mathcal{B}^{(1)}$ has self-intersection -1 in $Z^{(1)}$ and does not intersect the images by $q^{(1)} \circ q^{\prime}$ of the $\Gamma_{j}, j \in J$, otherwise it would have multiplicity $\geq 2$ in the fiber $\varphi^{(1)^{-1}}(\infty)$ and any component which have been contracted on it before would have originally multiplicity $\geq 2$ in $\varphi^{-1}(\infty)$.

Then, we can contract $q^{(2)}: Z^{(1)} \rightarrow Z^{(2)}$ all the extremities of $\mathcal{B}^{(1)}$ which have self-intersection -1 in $Z^{(1)}$.

By induction on the length of $\mathcal{B}^{(k)}$, we obtain that necessarily $J=\emptyset$.

In fact along the same lines we have also proved that, for $i=1, \ldots, r$, $n_{i}=1$, which gives our theorem.

\section{§2.2. $\quad$ Fiber over $\infty$}

The fiber of a compactification of a polynomial over $\infty$ is in general multiple.

Proposition 2.3. Let $\pi: Z \rightarrow \mathbb{P}^{2}$ be a modification of $\mathbb{P}^{2}$ which lifts the indeterminacy of the rational function $F / T^{d}$ associated to a polynomial function $f: \mathbb{C}^{2} \rightarrow \mathbb{C}$. The multiplicity of the strict transform $D_{\infty}$ of the line at infinity $T=0$ of $\mathbb{C}^{2}$ by $\pi$ equals the degree $d$ of $f$.

When the degree is $\geq 2$, the fiber $\varphi^{-1}(\infty)$ over $\infty$ of a minimal compactification $\varphi$ is obtained from the fiber $\varphi_{1}^{-1}(\infty)$ over $\infty$ of the contraction $\varphi_{1}$ given by Proposition 1.6 by a sequence of point blowing-ups. One starts by blowing-up a point of this fiber, say $C$, of self-intersection 0 , which becomes a non-singular curve of self-intersection -1 intersected transversally by the new exceptional divisor of self-intersection -1 . Then, we have to blow-up the intersection of these two non-singular curves of self-intersection -1 , because the fiber over $\infty$ of the minimal compactification of $f$ has only one component of self-intersection -1 . Now to continue we have to blow-up points over this last curve which has multiplicity 2 in the divisor inverse image of the original curve $C$, so that all the following curves that appear in the successive blowing-ups must have multiplicity $\geq 2$. As a consequence, we have: 
Proposition 2.4. Let $f$ be a rational polynomial. Let $\pi: Z \rightarrow \mathbb{P}^{2}$ be a modification of $\mathbb{P}^{2}$ on which $f$ extends to a map $\varphi: Z \rightarrow \mathbb{P}^{1}$. Assume it is a minimal compactification of $f$. Then in the fiber $\varphi^{-1}(\infty)$ of $\varphi$ over $\infty$, there are only two components with multiplicity 1.

Let $\pi: Z \rightarrow \mathbb{P}^{2}$ be a minimal modification of $\mathbb{P}^{2}$ on which $f$ extends to a map $\varphi: Z \rightarrow \mathbb{P}^{1}$. Let $q: Z \rightarrow Z_{1}$ be the contraction of Proposition 1.6. Now, since there is only one component of self-intersection -1 in the fiber $\varphi^{-1}(\infty)$ of the minimal compactification $\varphi$ of the rational polynomial $f, \varphi^{-1}(\infty)$ is the desingularization divisor of an analytic branch $(\Gamma, x)$ in $Z_{1}$ at a point $x \in \varphi_{1}^{-1}(\infty)$. Namely, if $(L, y)$ is the germ of a non-singular disc transversal to the strict transform $D_{\infty}$ of $T=0$, the line at infinity of $\mathbb{C}^{2}$, at a general point of $D_{\infty}$, one can choose $(\Gamma, x)$ as the image of $(L, y)$ by $q$.

One obtains $\varphi^{-1}(\infty)$ by starting with the blowing-up of the point $x$. Let $D_{0}$ be the strict transform of $\varphi_{1}^{-1}(\infty)$ by $q$. The intersection tree of the closure of $\varphi^{-1}(\infty) \backslash D_{0}$ is the intersection tree of a branch desingularization, namely the intersection tree of the desingularization of $(\Gamma, x)$ in this case. One can observe that with this understanding of the geometry, the degree $d$ coincide with the intersection multiplicity of $\varphi_{1}^{-1}(\infty)$ and $(\Gamma, x)$ at the point $x$ which is equal to a multiple of the multiplicity of $(\Gamma, x)$ or to the maximal contact number of $(\Gamma, x)$ with a non-singular curve at $x$.

This observation shows us that the intersection tree of $\varphi^{-1}(\infty)$ is of one of the following types:

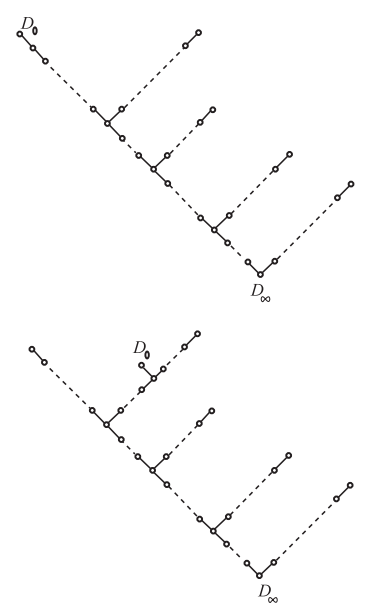

Figure 1. Intersection tree of $\varphi^{-1}(\infty)$ 
The first type correspond to the case the image $(\Gamma, x)$ of $(L, y)$ by $q$ has maximal contact with $\varphi_{1}^{-1}(\infty)$. The second type when it has no maximal contact.

\section{$\S 3 . \quad$ The Jacobian Conjecture}

In this section we shall show that if the polynomial $f$ belongs to a Jacobian pair and is a simple rational polynomial, it is up to composition with an automorphism of $\mathbb{C}^{2}$ a coordinate function.

\section{$\S 3.1$. Simple rational polynomials}

Let us suppose that after composition with any automorphism of $\mathbb{C}^{2}$ the polynomial $f$ is not a coordinate function. In [7] we have observed that it is equivalent to say that $f$ is not a locally trivial fibration over $\mathbb{C}$.

We have seen above that there is a modification $\pi: Z \rightarrow \mathbb{P}^{2}$ of $\mathbb{P}^{2}$, a sequence of point blowing-ups over the asymptotic directions of $f$, on which $f$ extends into a map $\varphi: Z \rightarrow \mathbb{P}^{1}$, called a compactification of $f$. We have seen that

$$
\pi^{-1}(T=0)=\mathcal{D}=Z \backslash \pi^{-1}\left(\mathbb{C}^{2}\right) .
$$

In the divisor $\mathcal{D}$ we have distinguished three types of components, the ones over which the restriction of $\varphi$ is constant with value $\infty$, the ones over which $\varphi$ has a constant value $\lambda \neq \infty$, the ones over which $\varphi$ is not constant and induces a map over $\mathbb{P}^{1}$ with degree $\geq 1$ that we have called dicritical components of $f$. The degree of the restriction of $\varphi$ to a dicritical component is called the degree of the dicritical component.

In this section we shall assume that $f$ is simple (see [10]), i.e. for all these dicritical components the restrictions of the compactification $\varphi$ of $f$ have degree 1. In [10] one can find a classification up to right-left equivalence of simple rational polynomials.

We have assumed that $f$ is not a locally trivial fibration over $\mathbb{C}$. Then, it means that at least one of the dicritical components of $f$ is non-equisingular. Recall that a dicritical component of $f$ is non-equisingular in the minimal compactification $Z_{0}$ of $f$ (see [7]) if, either the degree of this dicritical component of $f$ is $>1$, or it has degree one and it is intersected by the components at infinity contained in the inverse image by the compactification $\varphi$ of an atypical value $\lambda \in \mathbb{C}$ of $f$. A dicritical component of $f$ in a compactification $Z$ is non-equisingular if its image in a minimal compactification is non-equisingular in the preceding sense. 
Above we have studied non-general fibers of a minimal compactification of a rational polynomial which belongs to a Jacobian pair. We found that such fibers are reduced normal crossing divisors (see Theorem 2.1).

Observe that in this latter case the bamboo of non-singular rational curves, components of the divisor at infinity $\mathcal{D}$ and intersecting the non-equisingular dicritical component, is certainly not empty. Otherwise the non-general fiber of $\varphi$ would have an ordinary quadratic singularity at a point of the dicritical component. An immediate application of Rouché Theorem would show that the degree of that dicritical could not be 1 as supposed.

Let us summarize our observations in the following

Proposition 3.1. All the fibers over finite values of the minimal compactification of a simple rational polynomial which belong to a Jacobian pair are reduced and intersect dicritical component of the polynomial transversally. Non-general fibers over atypical values of the polynomial are divisors with normal crossings whose components at $\infty$ have self-intersection $\leq-2$.

\section{§3.2. Main result}

We shall prove:

Theorem 3.2. $\quad$ A simple rational polynomial which is not a locally trivial fibration over $\mathbb{C}$ cannot belong to a Jacobian pair.

This theorem shows in particular that the polynomial $f=X(X Y-1)$ given above as example cannot belong to a Jacobian pair, because we saw that, it is a rational polynomial, it is not a locally trivial fibration over $\mathbb{C}$ and one can prove that $f$ is a simple rational polynomial.

Proof. Assume that the simple rational polynomial $f$ is not a locally trivial fibration over $\mathbb{C}$. Then, as we already said above, one of its dicritical component is non-equisingular in the sense of [7, p. 379].

Let $\pi: Z \rightarrow \mathbb{P}^{2}$ be a modification of $\mathbb{P}^{2}$ on which $f$ has a compactification $\varphi: Z \rightarrow \mathbb{P}^{1}$.

Consider the canonical divisor $K_{Z}$ of $Z$. The components of the divisor $\pi^{-1}(T=0)=\mathcal{D}$ give a basis of the free abelian group $H_{2}(Z)$, the multiplicities of $K_{Z}$ along these components are well defined. A dicritical component of $f$ is said negative in $Z$ if its multiplicity in $K_{Z}$ is $<0$, it is said positive if its multiplicity in $K_{Z}$ is $\geq 0$. 
Remark 3.3. Observe that if $D$ is a dicritical component of $f$ in a modification $\pi: Z \rightarrow \mathbb{P}^{2}$ of $\mathbb{P}^{2}$, it is the strict transform in $Z$ of a dicritical component of $f$ in the minimal modification on which $f$ has a compactification. It is easy to see that the notion of positivity or negativity does not depend on the compactification of $f$ chosen (see below).

We have supposed that the simple rational polynomial $f$ belongs to a Jacobian pair $(f, g)$. We may assume that $Z$ is a modification of $\mathbb{P}^{2}$ on which $g$ has also a compactification $\psi: Z \rightarrow \mathbb{P}^{1}$. The Jacobian hypothesis implies that the support of the differential $\omega:=d \varphi \wedge d \psi$ is in $\mathcal{D}$. The canonical divisor $K_{Z}$ is given by this 2 -form $\omega$ which comes from the 2 -form $d x \wedge d y$ of $\mathbb{C}^{2}$. Therefore, along a negative dicritical component $D$ of $f$ in $Z$, the differential $\omega$ has a pole. Since $\varphi$ along $D$ defines a non-constant function, $\psi$ has a pole along $D$. In particular this implies :

Lemma 3.4. If a pair $(f, g)$ is a Jacobian pair, a negative dicritical for $f$ has multiplicity $\leq-2$ in the canonical divisor on modification of $\mathbb{P}^{2}$ on which $f$ extends.

Proof. Given a component at infinity $E$ of a modification $\pi: Z \rightarrow \mathbb{P}^{2}$ of $\mathbb{P}^{2}$, we know that it has a multiplicity in the canonical divisor $K_{Z}$ of $Z$. By point blowing-up the strict tranform of this component keep the same multiplicity in the new canonical divisor (see Proposition 3.3. of $\S 3$ Chapter $\mathrm{V}$ of [6]). So in any modification of $Z$, since it is obtained by composing a finite sequence of point blowing-ups, the strict transform of $E$ will keep the same multiplicity in the canonical divisor of the modification of $Z$.

In particular to have the multiplicity of a dicritical component of $f$ when it belongs to a Jacobian pair $(f, g)$, instead of computing it in the minimal compactification of $\mathbb{C}^{2}$ on which $f$ extends, it is enough to make the calculation in a modification of $\mathbb{P}^{2}$ on which both $f$ and $g$ extends as $\varphi$ and $\psi$. Since the pair $(f, g)$ is a Jacobian pair, the pole order of the differential form $d \varphi \wedge d \psi$ on the negative dicritical of $f$ is equal to the multiplicity that we are looking for. Then $\psi$ must have a pole on the negative dicritical component of $f$ in any $\mathbb{P}^{2}$ on which both $f$ and $g$ extends. This shows that the order of the pole of $d \varphi \wedge d \psi$ is $\leq-2$, as stated in the lemma.

Then,

Lemma 3.5. Let $f$ be a simple rational polynomial which belongs to a Jacobian pair. A negative dicritical component $D$ of $f$ is equisingular. 
In fact, we shall show the following result:

Lemma 3.6. Let $D$ be a negative dicritical component of $f$ along which $\psi$ is constant. If $D$ is non-equisingular, the pair $(f, g)$ is not a Jacobian pair.

Proof. Assume first that $D$ is a dicritical component of $f$ in a minimal modification $\pi_{0}: Z_{0} \rightarrow \mathbb{P}^{2}$ of $\mathbb{P}^{2}$ which lifts the indeterminacy of the rational function $F / T^{d}$ associated to $f$. Let $\varphi_{0}$ be the extension of $f$ to $Z_{0}$. Since $D$ is non-equisingular, by definition (see [7]) there is $\lambda \in \mathbb{C}$, such that either the fiber $\varphi_{0}^{-1}(\lambda)$ is reduced at a point $x \in D \cap \varphi^{-1}(\lambda)$ and has a critical point at $x$ or the fiber $\varphi_{0}^{-1}(\lambda)$ contains a bamboo of $\mathcal{D}$ which intersect $D$ at $x \in D$. Proposition 3.1 shows that, for a simple rational polynomial, we only have the second case.

Assume first that the lifting $G / T^{d^{\prime}} \circ \pi_{0}$ to $Z_{0}$ of the rational function $G / T^{d^{\prime}}$ defined by $g$ on $\mathbb{P}^{2}$ has no indeterminacy points on $A:=\left(D \backslash \mathcal{D}_{\infty}\right) \cup_{E \in \mathcal{B}} E$, where $\mathcal{B}$ is the bamboo of components at infinity contained in $\varphi_{0}^{-1}(\lambda)$ intersecting $D$.

Since $D$ is a negative dicritical component of $f$, the strict transform of $D$ in any modification $Z$ of $Z_{0}$, remains negative in the canonical divisor. This negativeness of the strict transform of $D$ in a modification over which $g$ has also a compactification means that the differential form $\omega$ defined by the extensions of $f$ and $g$ has a pole along the strict transform of $D$, i.e. the extension of $g$ has a pole along that component since the strict transform of $D$ is a dicritical component of $f$. In particular it means that the lifting $G / T^{d^{\prime}} \circ \pi_{0}$ to $Z_{0}$ has value $\infty$ on $D$.

Then, either the polynomial $g$ does not have any dicritical components contained in $D \cup_{E \in \mathcal{B}} E$, or $g$ has a dicritical components contained in $D \cup_{E \in \mathcal{B}} E$.

In the first case the lifting $G / T^{d^{\prime}} \circ \pi_{0}$ to $Z_{0}$ has value $\infty$ on the whole $A$. By contracting the bamboo $\cup_{E \in \mathcal{B}} E$, the components of which have selfintersection $\leq-2$, we obtain a normal surface singularity $\left(S, x_{1}\right)$ and, since the contracted bamboo of components is non-empty, this surface singularity is Jung-Hirzebruch, i.e. it is obtained as quotient of $\mathbb{C}^{2}$ by the proper and discrete action of a finite cyclic group (see [1] or [2]).

The functions $\varphi_{0}$ and $G / T^{d^{\prime}} \circ \pi_{0}$ induce functions $\varphi_{1}$ and $\psi_{1}$ on $\left(S, x_{1}\right)$. The map $\left(\varphi_{1}, \psi_{1}\right):\left(S, x_{1}\right) \rightarrow\left(\mathbb{P}^{1} \times \mathbb{P}^{1},(\lambda, \infty)\right)$ is finite, so it has a discriminant space $\Delta$ (see e.g. [11]). Let $u$ and $v$ be the coordinates in $\mathbb{P}^{1} \times \mathbb{P}^{1}$ at $(\lambda, \infty)$ which correspond to the respective values of $\varphi_{1}$ and $\psi_{1}$. At $(\lambda, \infty)$ this discriminant eventually contains the line $\left\{v=\psi_{1}(x)\right\}$. The discriminant $\Delta$ contains the line $\left\{v=\psi_{1}(x)\right\}$, but it also contains other components otherwise $\left(S, x_{1}\right)$ is nonsingular at $x_{1}$ (see e.g. [1]) which contradicts the fact that $\left(S, x_{1}\right)$ is obtained 
by contracting a non-empty bamboo of components of self-intersection $\leq-2$. Therefore the critical space of $\left(\varphi_{1}, \psi_{1}\right)$ contains components outside the image of the divisor at infinity $\mathcal{D}$ by the contraction which defines $\left(S, x_{1}\right)$. It is easy to see that this shows that on the modification $Z$ of $\mathbb{P}^{2}$ on which both $f$ and $g$ extend as $\varphi$ and $\psi$, the critical locus of $(\varphi, \psi)$ contains components outside of the divisor at $\infty$, which means that $(f, g)$ is not a Jacobian pair.

In the second case, since $D$ is a negative dicritical of $f$, the value of $G / T^{d^{\prime}}$ 。 $\pi_{0}$ at the general point of $D$ is $\infty$ and $D$ is not a dicritical component of $g$, so the dicritical component of $g$ is contained in $\varphi_{0}^{-1}(\lambda)$. Since $\varphi_{0}$ has a finite value $\lambda$ along that dicritical component of $g$, this dicritical component is positive, but this is impossible, because all the multiplicities of the canonical divisor of $Z_{0}$ on the components of $\mathcal{D}$ contained in $\varphi_{0}^{-1}(\lambda)$ are negative as the following lemma indicates:

Lemma 3.7. Let $X$ be a modification of $\mathbb{P}^{2}$. Let $\mathcal{D}$ be the divisor at $\infty$ of $X$. Let $\mathcal{B}=\left\{D_{1}, \ldots, D_{\ell}\right\}$ be a bamboo of components contained in $\mathcal{D}$. Assume that

1. the extremity $D_{1}$ of $\mathcal{B}$ does not intersect any other component of $\mathcal{D}$ but $D_{2}$,

2. no other component but $D_{\ell}$ in $\mathcal{D}$ intersect the sub-bamboo $\mathcal{B}^{\prime}=\left\{D_{1}, \ldots, D_{\ell-1}\right\}$

3. the self-intersections of $D_{1}, \ldots, D_{\ell-1}$ are $\leq-2$.

Then $D_{\ell}$ is negative (resp. positive) in the canonical divisor of $X$ if and only if $D_{1}$ is negative (resp. positive) in the canonical divisor of $X$.

Proof. The proof of this lemma is quite easy and only use Riemann-Roch Theorem on the surface $X$. Let $K_{X}$ be the canonical divisor of $X$ :

$$
K_{X}=m_{1} D_{1}+m_{2} D_{2}+\ldots+m_{\ell} D_{\ell}+\ldots
$$

Since all the curves $D_{i}$ are rational and non-singular we have

$$
K_{X} \cdot D_{i}+D_{i} \cdot D_{i}+2=0,
$$

for $1 \leq i \leq \ell-1$. We have:

$$
m_{2}+2=-\left(m_{1}+1\right)\left(D_{1} \cdot D_{1}\right) .
$$

Since we have assumed that $\left(D_{i} . D_{i}\right) \leq-2$ for $1 \leq i \leq \ell-1$, we obtain

$$
m_{2} \geq m_{1} \geq 0 \text { if } m_{1} \geq 0 \text { and } m_{2}<m_{1} \text { if } m_{1}<0 .
$$

For $2 \leq i \leq \ell-1$, we have:

$$
m_{i-1}+m_{i}\left(D_{i} \cdot D_{i}\right)+m_{i+1}+D_{i} \cdot D_{i}+2=0,
$$


which yields $m_{i+1}=-\left(m_{i}+1\right)\left(D_{i} . D_{i}\right)-m_{i-1}-2$. Since $-\left(D_{i} \cdot D_{i}\right) \geq 2$, for $1 \leq i \leq \ell-1$, we see that $m_{i} \geq m_{i-1} \geq 0$ implies $m_{i+1} \geq m_{i}$ and $m_{i}<m_{i-1}<0$ implies $m_{i+1}<m_{i}<0$.

This gives our lemma.

The above lemma shows that all the components $(E)_{E \in \mathcal{B}}$ of $\mathcal{D}$ contained in the bamboo $\mathcal{B}$ are negative in the canonical divisor of $Z_{0}$ if $D$ is negative, so $g$ cannot have a dicritical among them if $(f, g)$ is a Jacobian pair.

To finish the proof of Lemma 3.5 it remains to consider the case $G / T^{d^{\prime}} \circ \pi_{0}$ has points of indeterminacy on $A$.

If the points of indeterminacy are on $A \backslash \varphi_{0}^{-1}(\lambda)$ the preceding reasoning shows that the non-equisingularity of the dicritical component $D$ of $f$ implies that $f$ cannot belong to a Jacobian pair.

Therefore, we assume that the points of indeterminacy of $G / T^{d^{\prime}} \circ \pi_{0}$ lie on $\cup_{E \in \mathcal{B}} E$. It implies that on the minimal modification $Z$ of $\mathbb{P}^{2}$ on which both $f$ and $g$ extends as functions $\varphi$ and $\psi$, in $\varphi^{-1}(\lambda)$ some components are dicritical components of $g$. Since, as above these dicritical components of $g$ are necessarily positive, none of them are among the components which are strict transform of the components of $\varphi_{0}^{-1}(\lambda)$.

Let $q: Z \rightarrow Z_{0}$ the sequence of point blowing-ups which factorizes $\pi$ through $\pi_{0}$. Since the dicritical components of $g$ which lie in $\varphi^{-1}(\lambda)$ are positive, among the point blowing-ups of the sequence $q$ there are blowing-up of points which are not at the intersection of two components because one can find easily (using e.g. Proposition 3.3 of chap. V in [6]) that, blowing-up the intersection point of two negative components of multiplicities $m_{i}<0$ and $m_{j}<0$ in the canonical divisor, the exceptional divisor which appears has multiplicity $m_{i}+m_{j}+1<0$ in the new canonical divisor. This implies that the intersection tree of the components of $\varphi^{-1}(\lambda) \cap q^{-1}\left(\cup_{E \in \mathcal{B}} E\right)$ on which $\psi=\infty$ is not a bamboo, except in a very particular case that we shall consider later below. Since $Z$ is the minimal compactification for both $f$ and $g$, the components of this tree have self-intersection $\leq-2$. By contracting the divisor given by the components of this tree, one obtains a normal surface singularity $\left(S, y_{1}\right)$ which is not Jung-Hirzebruch.

The morphisms $\varphi$ and $\psi$ define functions $\varphi_{S}:\left(S, y_{1}\right) \rightarrow\left(\mathbb{P}^{1}, \lambda\right)$ and $\psi_{S}:$ $\left(S, y_{1}\right) \rightarrow\left(\mathbb{P}^{1}, \infty\right)$. The mapping $\left(\varphi_{S}, \psi_{S}\right):\left(S, y_{1}\right) \rightarrow\left(\mathbb{P}^{1} \times \mathbb{P}^{1},(\lambda, \infty)\right)$ is finite and has a discriminant $\Delta_{S}$. Considering the coordinates $u, v$ of $\left(\mathbb{P}^{1} \times \mathbb{P}^{1}\right)$ at $(\lambda, \infty)$ values of $\varphi_{S}$ and $\psi_{S}$ respectively, the set $\{u=\lambda\} \cup\{v=\infty\}$ is eventually contained in $\Delta_{S}$. Since $\left(S, y_{1}\right)$ is not a Jung-Hirzebruch singularity, the discriminant $\Delta_{S}$ contains other components than $\{u=\lambda\} \cup\{v=\infty\}$, so 
that $(f, g)$ cannot be a Jacobian pair.

It remains to consider the exceptional case when the intersection tree of the components of $\varphi^{-1}(\lambda) \cap q^{-1}\left(\cup_{E \in \mathcal{B}} E\right)$ on which $\psi=\infty$ could be a bamboo. This case may happen when the points of indeterminacy of $G / T^{d^{\prime}} \circ \pi_{0}$ on $\mathcal{E}:=\cup_{E \in \mathcal{B}} E$ are non-singular points of $\mathcal{E}$ and lie on a component which gives an endpoint of the bamboo $\mathcal{B}$ or a component of multiplicity -1 , say $E_{0}$, in the canonical divisor of $Z_{0}$ and the dicritical components of $g$ in $Z$ over these points are obtained by a single blowing-up. In the first case the indeterminacy of $G / T^{d^{\prime}} \circ \pi_{0}$ is lifted by a sequence of point blowing-ups and the corresponding dicritical component of $g$ is equisingular. We contract as above the divisor of $Z$ given by the tree $\mathcal{E}$, which is now a bamboo, but the multiplicity of $\varphi$ being one on the components of this bamboo, the discriminant $\Delta_{S}$ contructed as above does not contain the component $\{u=\lambda\}$, since it cannot be equal to $\{v=\infty\}$ alone, the critical space has points which are not at $\infty$ and $(f, g)$ cannot be a Jacobian pair.

In summary, if $(f, g)$ is a Jacobian pair, the negative dicritical $D$ of $f$ has to be equisingular.

End of Proof of the main Theorem. In the Proposition 2.4 we have noticed that the fiber over $\infty$ of the minimal compactification of a rational polynomial has only two components of multiplicity 1 . Since the degree of a dicritical component of a polynomial equals the multiplicity of the component of the divisor defined by the fiber over infinity of the minimal compactification that it intersects, the dicritical components of a simple rational polynomial intersect one of the two components of multiplicity one in the fiber over infinity of the minimal compactification.

We resume the notations used above for the minimal compactification $\varphi_{0}$ : $Z_{0} \rightarrow \mathbb{P}^{1}$ of the simple rational polynomial $f$. In the case of a simple rational polynomial the situation is very particular, because when the simple rational polynomial is not a fibration on $\mathbb{C}$, to obtain $Z_{0}$, we have to blow-up two points on the line $T=0$ at infinity of $\mathbb{C}^{2}$. In $Z_{0}$ over each of these points, there are dicritical components of $f$. These dicritical components of $f$ intersect the components of $\mathcal{D}_{\infty}$ which have multiplicity one, so there are only two possibilities:

1. either the intersection tree of $\varphi_{0}^{-1}(\infty)$ is a one Puiseux pair plane branch desingularization tree (see e.g. [12]) with a vertex attached to the branch of the desingularization tree whose extremity component has a curvette, i.e. germ at the intersection point with the component of a non-singular 
disc transverse to this component at a general point, whose image by $q$ has maximal contact with the considered one Puiseux pair curve, this vertex having the self-intersection equal to $-(k+1)$, where $k$ is the integral part of the Puiseux exponent; notice that the desingularization tree is the one of the branch $(\Gamma, x)$ of $\S 2.2$.

2. either the intersection tree of $\varphi_{0}^{-1}(\infty)$ is a bamboo $\mathcal{B}$ of $k(k \geq 2)$ nonsingular rational curves all of self-intersection -2 intersecting $D_{\infty}$, of selfintersection -1 , to which it is attached a self-intersection $-k$ non-singular rational curve, say $E$.

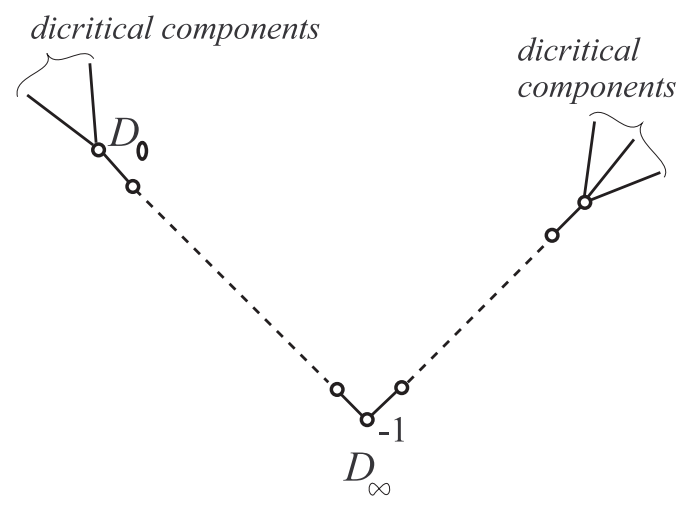

Figure 2. Intersection tree of $\varphi_{0}^{-1}(\infty)$

In the first case, intersecting the strict transform $D_{\infty}$ in $Z_{0}$ of the line at infinity $T=0$ of $\mathbb{C}^{2}$ are connected two bamboos of components of $\mathcal{D}_{\infty}=$ $\varphi_{0}^{-1}(\infty)$. If both of these bamboos contain a component of self-intersection $\leq-3$ in $Z_{0}$ which is not any of the extremities, the curvette of at least one dicritical component of $f$ for each bamboo has necessarily one Puiseux pair. This always happen except if the desingularization tree is the one of a curve with one Puiseux pair $\left(a_{1} a_{2}+1, a_{2}\right)$, i.e. a Puiseux exponent of type

$$
a_{1}+\frac{1}{a_{2}}
$$

where $a_{1}$ and $a_{2}$ are relatively prime integers.

When the image in $\mathbb{P}^{2}$ by $\pi_{0}$ of curvettes of dicritical components, i.e. germs at the intersection point with the dicritical component of non-singular discs transverse to the dicritical component at a general point, have one Puiseux pair, the corresponding dicritical components are non-equisingular. Using, for 
instance, Lemma 3.7 above, one can easily prove that at least one of these nonequisingular dicritical components of $f$ is negative, then Lemma 3.6 implies that $f$ cannot belong to a Jacobian pair.

In the case the intersection tree of $\varphi_{0}^{-1}(\infty)$ is the desingularization tree of a curve with one Puiseux pair $\left(a_{1} a_{2}+1, a_{2}\right)$, the tree is the following:

from the strict transform $D_{\infty}$ of the line at infinity $\{T=0\}$ which has selfintersection -1 start two bamboos, one, say $\mathcal{B}_{1}$, with $a_{2}-1(-2)$-self-intersection curves and an end-curve of self-intersection $-\left(a_{1}+1\right)$, the other one, say $\mathcal{B}_{2}$, with a $\left(-\left(a_{2}+1\right)\right)$-self-intersection curve intersecting $D_{\infty}$ followed by $a_{1}-1$ $(-2)$-self-intersection curves. In this case, if $a_{1}>1$ and $a_{2}>1$, there will be only one dicritical component of $f$ intersecting the endpoint of $\mathcal{B}_{2}$ and it is a negative dicritical component of $f$ which is non-equisingular.

If $a_{1}=1$ and $a_{2}>1$, all the self-intersections on the bamboo $\mathcal{B}_{1}$ are -2 , so there is only one dicritical component of $f$ intersecting this bamboo. It is easy to understand that this dicritical is equisingular and positive if $a_{2} \geq 3$. The non-equisingular dicritical has to intersect $\mathcal{B}_{2}$. Since for $a_{2} \geq 3$, the other dicritical component intersecting $\mathcal{B}_{1}$ is positive, so this dicritcal component intersecting $\mathcal{B}_{2}$ has to be negative and, by Lemma 3.6, $f$ does not belong to a Jacobian pair. If $a_{2}=2$, the dicritical component intersecting $\mathcal{B}_{1}$ is negative and equisingular, but its multiplicity in the canonical divisor is -1 which is excluded by Lemma 3.4 .

The case $a_{1}=1$ and $a_{2}=1$ is trivial.

In summary, a simple rational function which is not a locally trivial fibration over $\mathbb{C}$ cannot belong to a Jacobian pair. So, we have obtained:

Corollary 3.8. Let $(f, g)$ be a Jacobian pair. If $f$ is a simple rational polynomial, then $(f, g)$ is an algebraic automorphism of $\mathbb{C}^{2}$.

\section{References}

[1] W. Barth, C. Peters and A. Van de Ven, Compact complex surfaces, Ergebnisse der Mathematik und ihrer Grenzgebiete (3), Springer, Berlin, 1984.

[2] K. Behnke and O. Riemenschneider, Quotient surface singularities and their deformations, in Singularity theory (Trieste, 1991), 1-54, World Sci. Publ., River Edge, NJ, 1995.

[3] L. Eichenberger, Thèse de Doctorat, Inst. de Math., Univ. Genève, 1996.

[4] P. Griffiths and J. Harris, Principles of algebraic geometry, Wiley-Intersci., New York, 1978

[5] Hà Huy Vui and Lê Dũng Tráng, Sur la topologie des polynômes complexes, Acta Math. Vietnam. 9 (1984), no. 1, 21-32 (1985). 
[6] R. Hartshorne, Algebraic geometry, Graduate Texts in Mathematics, 52, Springer, New York, 1977.

[7] Lê Dũng Tráng and C. Weber, A geometrical approach to the Jacobian conjecture for $n=2$, Kodai Math. J. 17 (1994), no. 3, 374-381.

[8] _ Polynômes à fibres rationnelles et conjecture jacobienne à 2 variables, C. R. Acad. Sci. Paris Sér. I Math. 320 (1995), no. 5, 581-584.

[9] Lê Dũng Tráng, F. Michel and C. Weber, Sur le comportement des polaires associées aux germes de courbes planes, Compositio Math. 72 (1989), no. 1, 87-113.

[10] W. D. Neumann and P. Norbury, Rational polynomials of simple type, Pacific J. Math. 204 (2002), no. 1, 177-207.

[11] B. Teissier, The hunting of invariants in the geometry of discriminants, in Real and complex singularities (Proc. Ninth Nordic Summer School/NAVF Sympos. Math., Oslo, 1976), 565-678, Sijthoff and Noordhoff, Alphen aan den Rijn, 1977.

[12] O. Zariski, Algebraic surfaces, Second supplemented edition, with appendices by S. S. Abhyankar, J. Lipman, and D. Mumford. Ergebnisse der Mathematik und ihrer Grenzgebiete, 2. folge, Band 61, Springer-Verlag, New York, 1971. 